\section{Adhesion of surfaces mediated by adsorbed particles: Monte Carlo simulations and a general relationship between adsorption isotherms and effective adhesion energies}

\author{
Tillmann Stieger, ${ }^{a}$ Martin Schoen ${ }^{a b}$ and Thomas R. Weikl ${ }^{* c}$ \\ Received 3rd July 2012, Accepted 10th September 2012 \\ DOI: $10.1039 / \mathrm{c} 2 \mathrm{sm} 26544 \mathrm{c}$
}

In colloidal and biological systems, interactions between surfaces are often mediated by adsorbed particles or molecules that interconnect the surfaces. In this article, we present a general relationship between the adsorption isotherms of the particles and the effective, particle-mediated adhesion energies of the surfaces. Our relationship is based on the analysis and modeling of detailed data from Monte Carlo simulations. As general properties that should hold for a wide class of adsorption scenarios, we find (i) that the particle-mediated adhesion energies of surfaces are maximal at intermediate bulk concentrations of the particles, and (ii) that the particle coverage in the bound state of the surfaces is twice the coverage in the unbound state at these bulk concentrations.

\section{Introduction}

Adhesion and adsorption are important phenomena in both colloidal and biological systems. Characteristic aspects of these systems are that the constituent molecules or particles typically differ in size, and that the interactions between these constituents are often dominated by surface interactions. Adsorption refers to the binding of molecules or particles to the surfaces of larger constituents and is typically characterized by adsorption isotherms, i.e. by the surface concentrations of adsorbed molecules or particles as a function of their bulk concentration or chemical potential. Adhesion refers to the binding of two surfaces that are typically large compared to molecular dimensions and is characterized by adhesion energies per area.

Adsorption can lead to adhesion if molecules or particles bind to two apposing surfaces, e.g. to the surfaces of two larger particles or objects. The adhesion and aggregation of nanoparticles or microparticles, for example, can be mediated by adsorbed proteins ${ }^{1-5}$ or polymers. ${ }^{6-10}$ Nanoparticles can affect the adhesion of microparticles. ${ }^{11}$ The adhesion of lipid membranes can be caused by adsorbed proteins ${ }^{12,13}$ or multivalent ions ${ }^{14}$ that crosslink the membranes. Membrane adhesion may also be mediated by soluble proteins that interconnect receptor and ligand proteins anchored in apposing membranes. ${ }^{15,16}$

${ }^{a}$ Technische Universität Berlin, Stranski-Laboratorium für Physikalische und Theoretische Chemie, Straße des 17. Juni 115, 10623 Berlin, Germany ${ }^{b}$ North Carolina State University, Department of Chemical and Biomolecular Engineering, 911 Partners Way, Raleigh, NC 27695, USA ${ }^{c}$ Max Planck Institute of Colloids and Interfaces, Department of Theory and Bio-Systems, Science Park Golm, 14424 Potsdam, Germany
In this article, we consider an ensemble of particles between two parallel surfaces in Monte Carlo simulations. The two surfaces can be seen as surface segments in the contact zone of two constituents in colloidal or biological systems that are significantly larger than the particles. The particles adsorb on the surfaces and mediate adhesion if the separation of the surfaces is close to the diameter of the particles. In our Monte Carlo simulations, we determine the pressure that the particles exert on the surfaces and the area concentrations of the adsorbed particles at different surface separations. The effective particle-mediated adhesion energy of the surfaces is then obtained by integrating the pressure. Interestingly, the effective adhesion energy is maximal at intermediate bulk concentrations of the particles.

Our analysis of the Monte Carlo results indicates that the surface concentrations of the adsorbed particles depend in good approximation on a single parameter, the sum of the chemical potential and the binding energy of the particles, at least for binding energies that are significantly larger than the thermal energy $k T$ where $k$ is Boltzmann's constant and $T$ denotes the temperature. Integration of these surface concentrations, or adsorption isotherms, leads to free energies of adsorption in the bound and unbound state of the surfaces. These free energies of adsorption provide the basis for a simple model to calculate effective, particle-mediated adhesion energies of surfaces that can be generalized to a wide class of adsorption isotherms. The simple model is in good agreement with the effective adhesion energies obtained directly from the pressure measured in our Monte Carlo simulations. In addition, the model explains why the particle-mediated adhesion energies of surfaces are maximal at intermediate bulk concentrations of the particles, and why the particle coverage in the bound state of the surfaces is twice the coverage in the unbound state at these bulk concentrations. Our 
model generalizes and helps to understand previous results obtained in the special case of Langmuir adsorption. ${ }^{17,18}$

\section{The simulation model}

We consider spherical particles between two parallel surfaces (see Fig. 1). The particles repel each other, but are attracted by the surfaces. In our model, the interaction potential $V_{\mathrm{ps}}$ between the particles and the surfaces is short-ranged and decays to zero at separations $z$ of the particles from the surfaces close to the particle diameter $d$ (see Fig. 2). The interaction potential $V_{\mathrm{ps}}$ attains its minimum value $-U$ at the separation of $z=d / 2$ at which the particles are in close contact with the surfaces. The depth $U$ of the potential minimum corresponds to the binding energy of the particles at the surfaces. The soft, pairwise repulsion of the particles has the form $V_{\mathrm{pp}}=4 k T(d / r)^{12}$ where $r$ is the distance between two particle centers.

We assume that the two parallel surfaces are segments of colloidal objects that are large compared to the particles and surrounded by the particle solution. The number of particles between the parallel surfaces then varies because these particles can exchange with the surrounding bulk of particles. We further assume that the bulk particles constitute a large particle reservoir, with a bulk concentration $X_{\mathrm{b}}$ of particles that is determined by the chemical potential $\mu$ of the particles (see Fig. 3). The ensemble of particles between the two parallel surfaces considered here then corresponds to a grand-canonical ensemble with chemical potential $\mu$.

\section{Excess pressure and effective adhesion potential}

In this section, we determine the effective, particle-mediated adhesion potential of the surfaces from the pressure that the particles exert on the surfaces. This pressure depends on the separation $L$ of the surfaces and can be obtained from Monte Carlo simulations (see Appendix for details). We consider here the excess pressure to be

$$
\Delta p(L)=p(L)-p(L=\infty)
$$

since we assume that the two surfaces are surface segments of larger objects that are fully surrounded by the particles. Therefore at large separations, the overall forces exerted by the particles are zero.

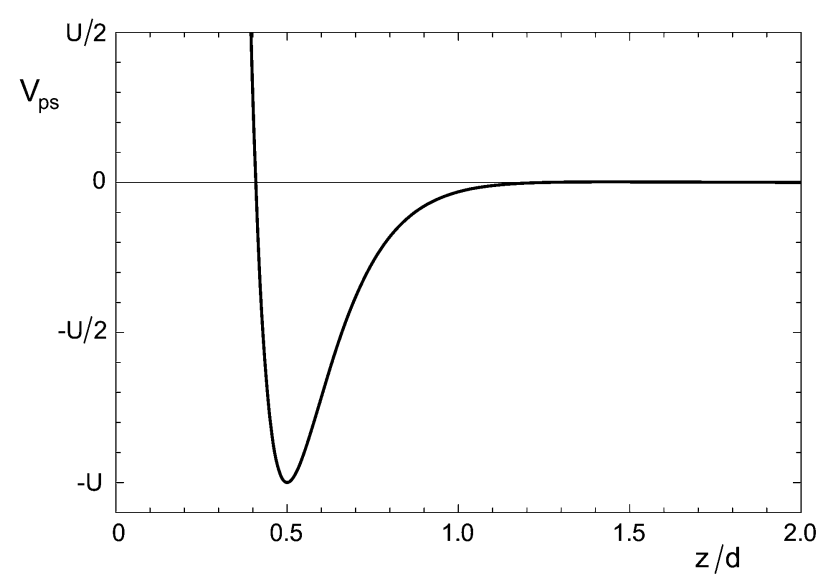

Fig. 2 The particle-surface interaction potential $V_{\mathrm{ps}}$ depends on the distance $z$ of the particle center from the surface. The interaction potential attains its minimum value $-U$ at the separation $z=0.5 d$ at which the spherical particle is in contact with the surface. The depth $U>$ 0 of the minimum is the binding energy of the particle with the surface. The potential is composed of a soft repulsive and a Yukawa-like attractive term (see eqn (12) and (13) in the Appendix section for details).

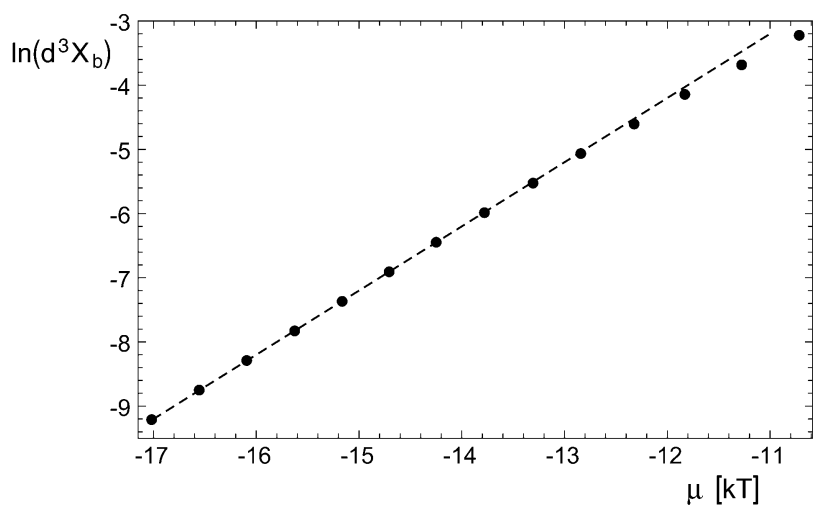

Fig. 3 Bulk concentration $X_{\mathrm{b}}$ versus chemical potential $\mu$ of the particles in our model (data points). At small values of $X_{\mathrm{b}}$ and $\mu$, the two quantities are related via $\ln \left(d^{3} X_{\mathrm{b}}\right) \simeq 7.8 k T+\mu$ (dashed line).

The effective, particle-mediated adhesion potential $V_{\text {ef }}$ of the surfaces is obtained by integration over the excess pressure $\Delta p(L)$ :

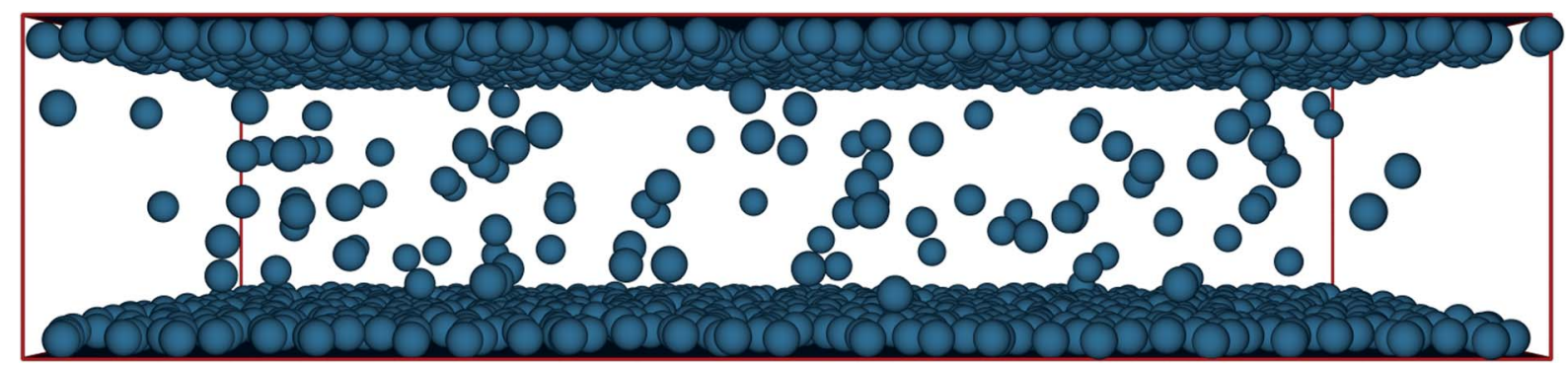

Fig. 1 Monte Carlo snapshot for the surface separation $L=10 d$ where $d$ is the diameter of the particles. The particles repel each other, but are attracted by the surfaces. In this snapshot, the chemical potential of the particles is $\mu=-12.32 k T$, which corresponds to a bulk concentration of $X_{\mathrm{b}}=0.01 / d^{3}$ of the particles away from the surfaces. The binding energy $U=10 \mathrm{kT}$ of the particles at the surfaces leads to an area concentration of $X_{\mathrm{s}}=0.42 / d^{2}$ of the particles in the adsorption layers. 


$$
V_{\mathrm{ef}}(L)=\int_{L}^{\infty} \Delta p\left(L^{\prime}\right) \mathrm{d} L^{\prime}
$$

In Fig. 4 , the excess pressure $\Delta p(L)$ and the effective adhesion potential $V_{\text {eff }}(L)$ are shown for the binding energy $U=10 \mathrm{k} T$ and chemical potential $\mu=-12.32 k T$, which corresponds to a bulk
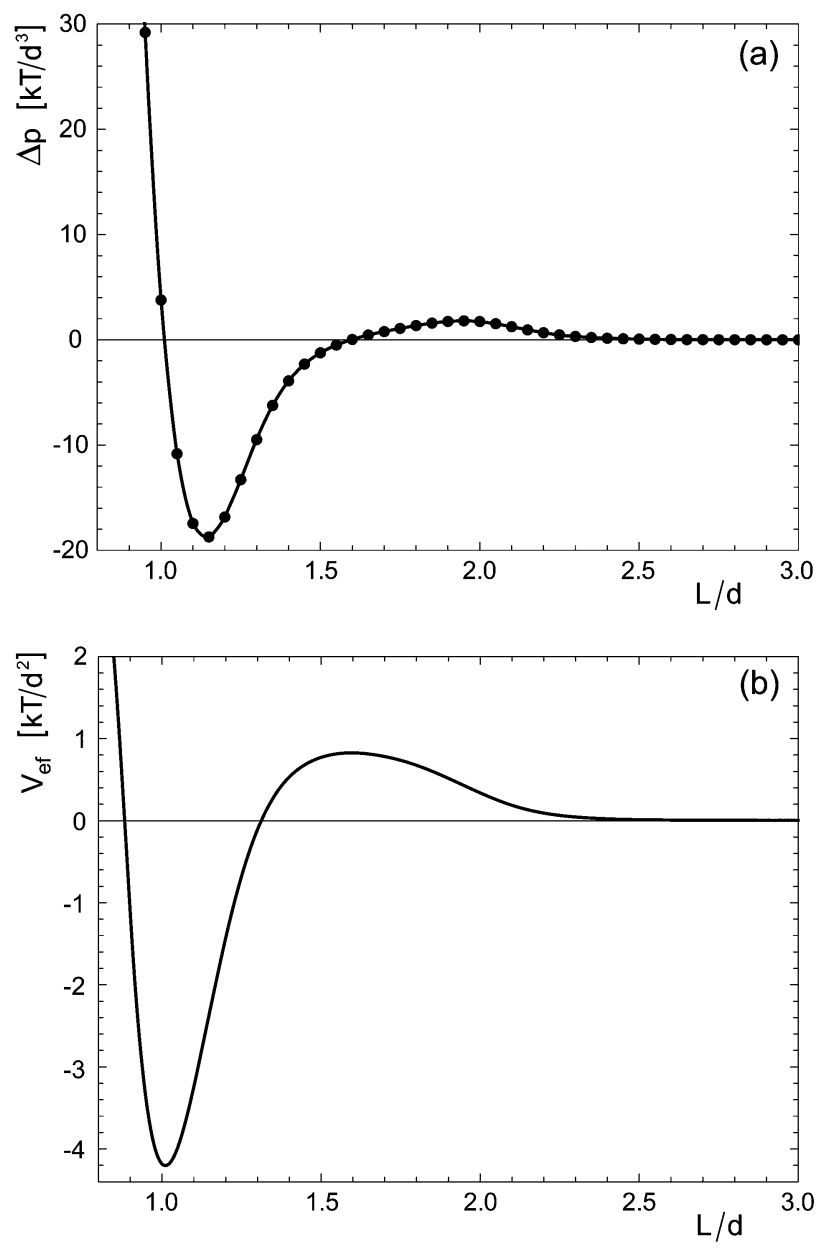

Fig. 4 (a) Excess pressure $\Delta p$ exerted by the particles and (b) effective, particle-mediated adhesion potential $V_{\text {ef }}$ of the surfaces as functions of the surface separation $L$ in units of the particle diameter $d$. In this example, the binding energy of the particles is $U=10 \mathrm{kT}$ and the chemical potential is $\mu=-12.32 k T$, which corresponds to a bulk particle concentration of $X_{\mathrm{b}}=0.01 / d^{3}$. The dots in subfigure (a) represent the Monte Carlo data, and the line results from interpolation. The effective potential $V_{\text {ef }}$ in subfigure (b) is obtained from the excess pressure $\Delta p$ via integration. The effective potential exhibits a minimum at a surface separation close to the particle diameter at which the particles are firmly bound to both surfaces. The depth $U_{\text {ef }}$ of the potential minimum is the effective binding of the surfaces. Because of the entropy of the confined particles, the minimum is located at a surface separation slightly larger than the separation $L=d$ where the total binding energy to both surfaces is minimal for a particle. In this example, the minimum is located at $L \simeq$ $1.01 d$, and the effective binding energy is $U_{\mathrm{ef}} \simeq 4.20 \mathrm{kT} / d^{2}$. The effective potential exhibits a barrier of height $U_{\mathrm{ba}}$ at intermediate separations at which particles that bind to one of the surfaces obstruct the binding of particles to the other surface. In this example, the barrier is located at $L \simeq 1.60 d$ and has the height $U_{\mathrm{ba}} \simeq 0.83 \mathrm{kT} / \mathrm{d}^{2}$.
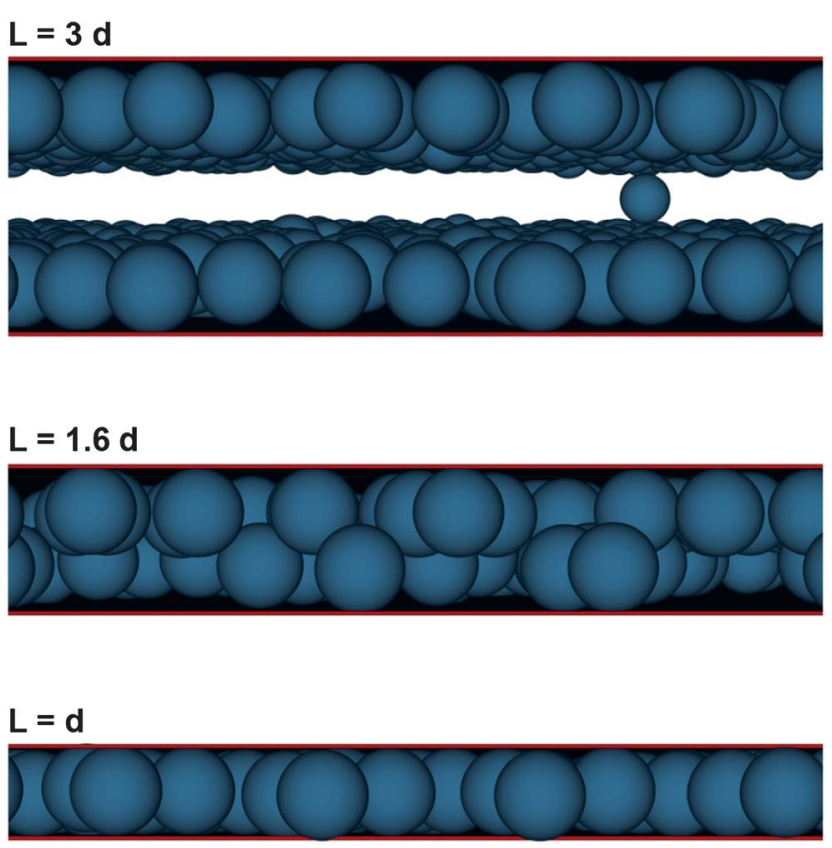

Fig. 5 Monte Carlo snapshots at the surface separations $L=3 d, 1.6 d$, and $d$ for the same parameters as in Fig. 4.

concentration $X_{\mathrm{b}}=0.01 / d^{3}$ of the particles. The effective adhesion potential exhibits a minimum value $-U_{\text {ef }}$ at surface separations $L$ close to the diameter $d$ of the particles since the particles can bind to both surfaces at this separation. The depth $U_{\text {ef }}$ of this minimum corresponds to the effective, particle-mediated adhesion energy of the surfaces. At surface separations $L$ around $1.6 d$, the effective adhesion potential $V_{\text {ef }}$ has a local maximum because particles can no longer bind to both surfaces, and because particles that bind to one of the surfaces sterically obstruct the binding of particles to the other surface (see Fig. 5 and 6). This maximum of height $U_{\mathrm{ba}}$ constitutes a barrier for adhesion. At larger surface separations $L \gtrsim 3 d$, the effective potential $V_{\text {ef }}$ decays to zero because the particles adsorb independently on the two surfaces.

A characteristic feature of the effective, particle-mediated adhesion energy $U_{\text {ef }}$ is that it exhibits a maximum at an intermediate value $\mu=\mu^{*}$ of the chemical potential (see Fig. 7). With increasing binding energy $U$ of the particles, the location of this maximum is shifted to smaller values of the chemical potential (see Fig. 8) and, thus, to smaller bulk concentrations $X_{\mathrm{b}}$ of the particles. In the following, we will show that the maximum of the function $U_{\mathrm{ef}}(\mu)$ can be understood from the adsorption isotherms and adsorption free energies of the particles. Our starting point is the surface concentration of adsorbed particles considered in the next section.

\section{Surface concentrations of particles}

The surface concentrations of the particles in the adsorption layers can be calculated from the concentration profiles $X(z)$ of the particles between the surfaces (see Fig. 6). Here, $z$ is the Cartesian coordinate perpendicular to the two surfaces, which are located at $z=0$ and $z=L$. For large surface separations $L \gtrsim$ $3 d$, the particle concentration $X(z)$ has two pronounced peaks 

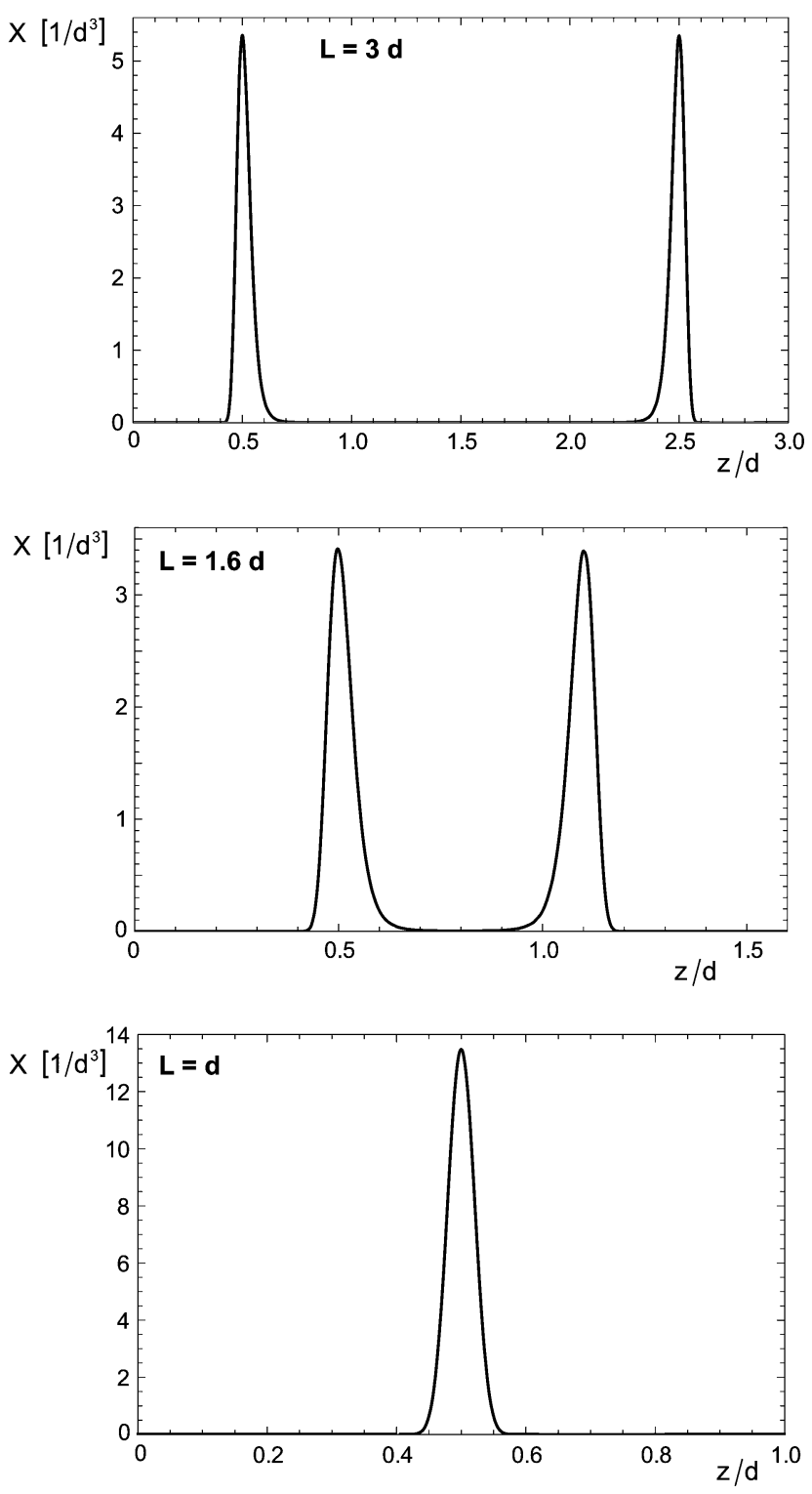

Fig. 6 Concentration profiles $X(z)$ of the particles between the surfaces for the same surface separations $L$ and parameters as in Fig. 5. The two peaks in the profiles for $L=3 d$ and $L=1.6 d$ correspond to the single layers of particles adsorbed at the two surfaces. The peaks at the separation $L=1.6 d$ are lower in height than the peaks at $L=3 d$ because particles that bind to one of the surfaces sterically obstruct the binding of particles to the other surface at this separation (see the snapshot in Fig. 5 for $L=1.6 d$ ). The single peak in the concentration profile at the separation $L=d$ corresponds to a layer of particles bound to both surfaces.

with maxima at $z$-values close to $0.5 d$ and $L-0.5 d$ where the particle-surface interaction potential $V_{\mathrm{ps}}$ is minimal. These two peaks correspond to the single layers of adsorbed particles at the two surfaces (see Fig. 5 and 6). At intermediate $z$-values in the range $d<z<L-d$ between the two peaks, the particle concentration $X$ tends towards the bulk concentration $X_{\mathrm{b}}$ because the particle-surface potential $V_{\mathrm{ps}}$ is practically 0 for these $z$-values, and because packing effects of the particles between the surfaces are negligible for the bulk concentrations $X_{\mathrm{b}}$ $<0.1 d^{3}$ considered here. For surface separations $L$ close to the

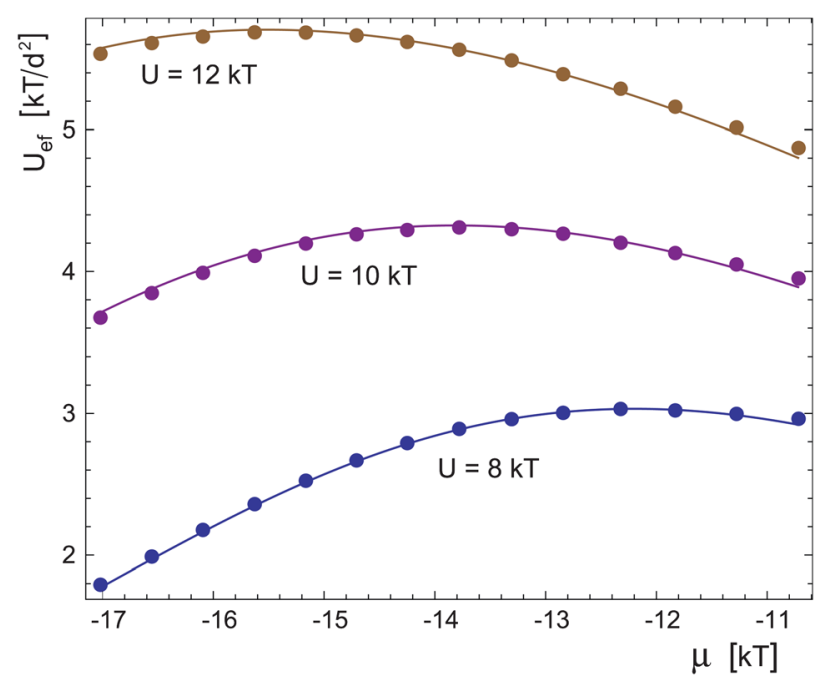

Fig. 7 Effective, particle-mediated binding energy $U_{\text {ef }}$ of the surfaces as a function of the chemical potential $\mu$ for the binding energies $U=8,10$, and $12 k T$ of the particles. The effective binding energy $U_{\text {ef }}$ exhibits a maximum at intermediate values of the chemical potential. The points represent the Monte Carlo data, and the lines the simple model based on eqn (5).

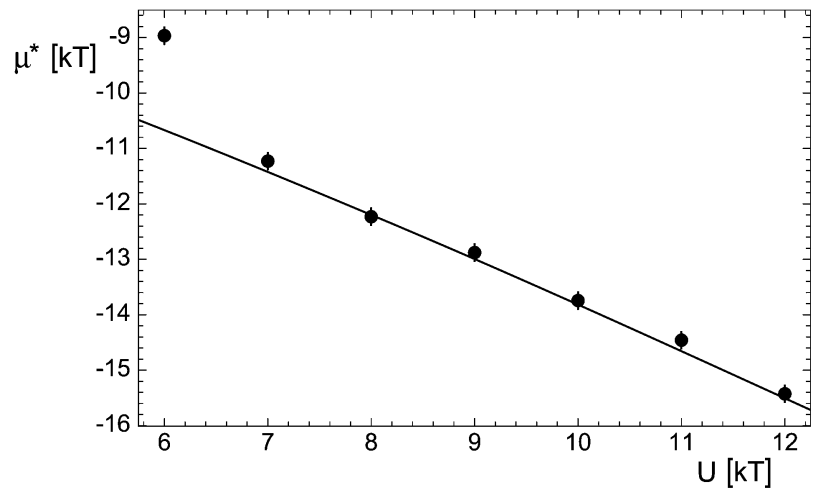

Fig. 8 Values $\mu^{*}$ of the chemical potential at which the effective binding energy $U_{\text {ef }}$ of the surfaces is maximal versus binding energy $U$ of the particles. The data points result from an interpolation of Monte Carlo data for $U_{\text {ef }}$ as a function of $\mu$ (see e.g. data points in Fig. 7). The line results from eqn (7) of the simple model with the fit function for the adsorption isotherm $X_{\mathrm{s}}\left(\mu+U_{L}\right)$ indicated as a dashed line in Fig. 9(b). The simple model is in good agreement with the Monte Carlo results for particle binding energies $U \geq 7 k T$, but deviates at smaller binding energies.

binding separation $d$, the concentration profile $X_{z}$ has a single peak that corresponds to a single layer of particles bound to both surfaces. The surface concentration $X_{\mathrm{s}}$ of particles in the adsorption layers is obtained by integration over the peaks in the concentration profiles $X(z)$ :

$$
X_{\mathrm{s}}=\int_{0}^{d} X(z) \mathrm{d} z
$$

For large surface separations, the surface concentration $X_{\mathrm{s}}$ defined in eqn (3) is the area concentration of the single layer of particles adsorbed to one of the surfaces. For the surface 
separation $L=d$, the surface concentration $X_{\mathrm{s}}$ is the area concentration of the particles that are bound to both surfaces.

In Fig. 9(a), the surface concentration $X_{\mathrm{s}}$ is shown as a function of the chemical potential $\mu$ at the binding separation $L=d$ of the surfaces and at the large surface separation $L=10 d$, for the three binding energies $U=8,10$, and $12 k T$ of the particles. The area concentration $X_{\mathrm{s}}$ increases with $\mu$ and with the binding energy $U$ of the particles, and is significantly larger at the surface separation $L=d$ because the particles bind to both surfaces. When plotted as a function of $\mu+U_{L}$ with $U_{L}=U$ for large $L$ and $U_{L}=2 U$ for $L=d$, the six curves of Fig. 9(a) fall onto a single curve (see Fig. 9(b)), which indicates (i) that the surface concentration $X_{\mathrm{s}}$ depends on the sum of the chemical potential and binding energy of the particles, and (ii) that the binding energy at the surface separation $L=d$ is approximately twice the binding energy at large separations, which is plausible since the
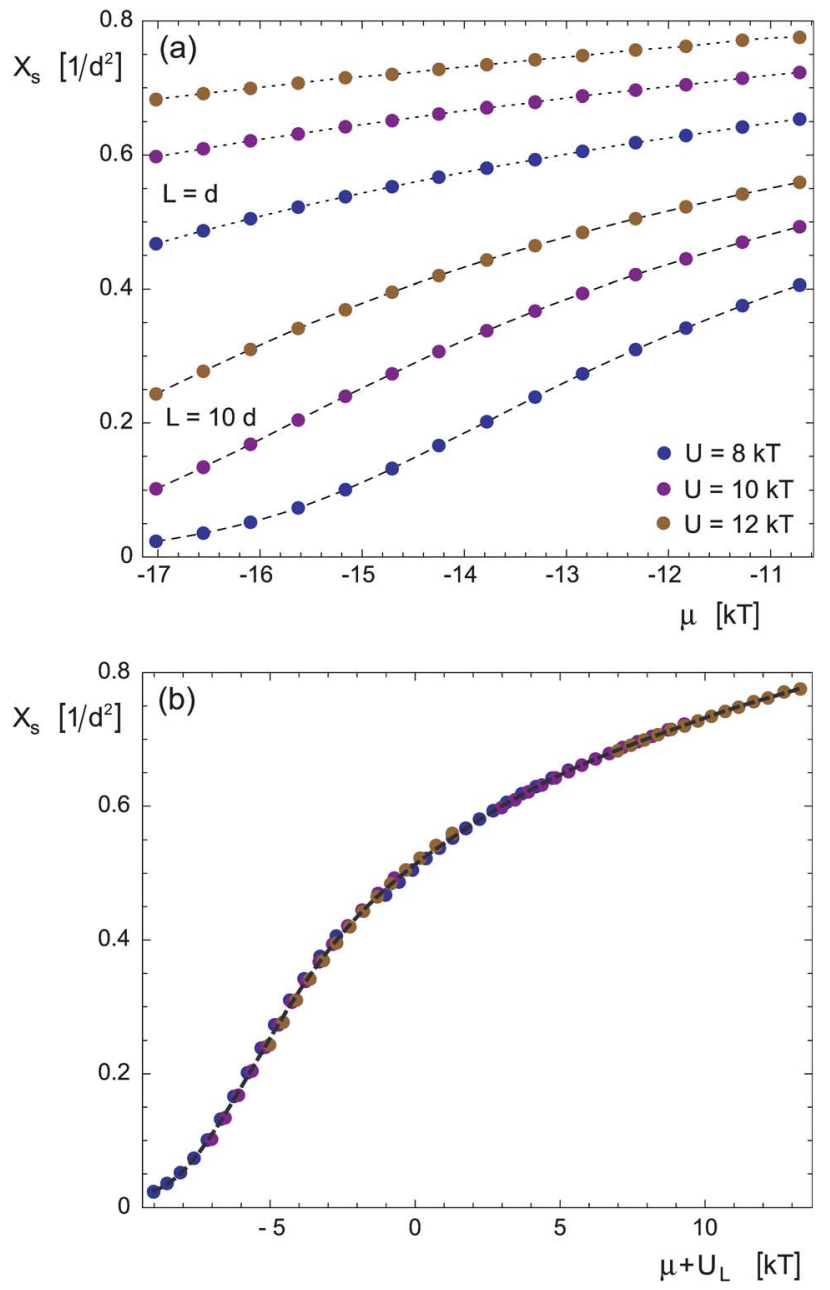

Fig. 9 (a) Surface concentration $X_{\mathrm{s}}$ of particles in the adsorption layers as a function of the chemical potential $\mu$ at the large surface separation $L=10 d$ (three bottom lines) and at the binding separation $L=d$ at which the particles strongly bind to both surfaces. At both separations, the surface concentration $X_{\mathrm{s}}$ increases with the chemical potential $\mu$ and with the binding energy $U$ of the particles. (b) Same surface concentrations $X_{\mathrm{s}}$ as a function of the rescaled chemical potential $\mu+U_{L}$ with $U_{L}=U$ for $L=10 d$ and $U_{L}=2 U$ for $L=d$. In this rescaled plot, the six curves of subfigure (a) fall onto a single curve. The dashed line represents a $9^{\text {th }}$ order polynomial fit to the Monte Carlo data (see Appendix).

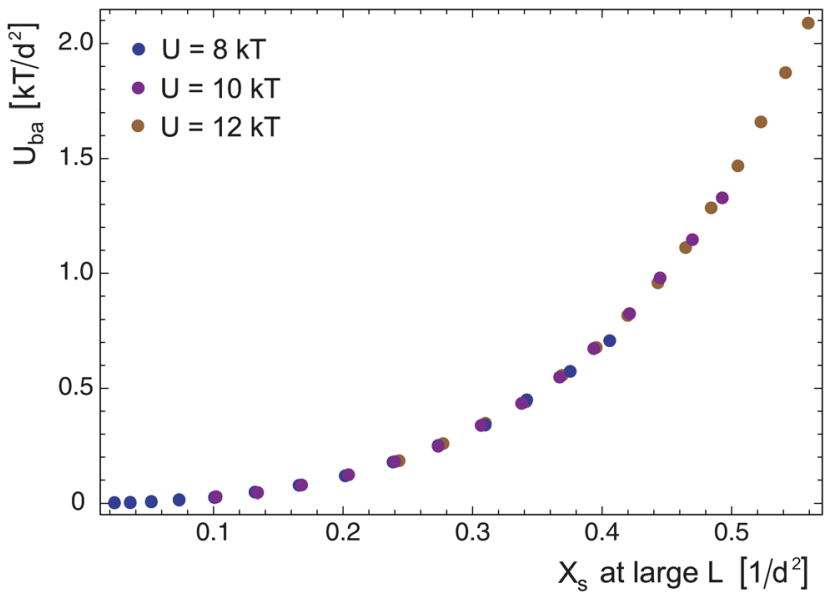

Fig. 10 Height $U_{\mathrm{ba}}$ of the barrier in the effective potential $V_{\text {ef }}$ as a function of the surface concentration $X_{\mathrm{s}}$ of adsorbed particles at the large surface separation $L=10 d$ for the binding energies $U=8,10$, and $12 k T$ of the particles.

particles bind to both surfaces at this separation. The small deviations between the curves in Fig. 9(b) presumably result from small differences in the entropies of bound particles at $L=d$ and at large $L$, which appear to be negligible compared to the binding energies, at least for the binding energies $U$ much larger than the thermal energy $k T$ considered here. Because of the soft repulsive interactions of the particles, the surface concentration $X_{\mathrm{S}}$ does not saturate at large values of $\mu+U_{L}$. A scaling argument indicates that $X_{\mathrm{s}}$ increases proportional to $\left(\mu+U_{L}\right)^{1 / 6}$ for large values of $\mu+U_{L}$ at which the adsorbed particles are arranged in a hexagonal lattice (see Appendix).

The surface concentration $X_{\mathrm{s}}$ of the particles at large surface separation determines the height $U_{\mathrm{ba}}$ of the barrier in the effective potential $V_{\text {ef }}$. In Fig. 10, the barrier height $U_{\mathrm{ba}}$ of the effective potential is shown as a function of this surface concentration for the three binding energies $U=8,10$, and $12 k T$ of the particles. The values of $X_{\mathrm{s}}$ here correspond to the values in Fig. 9(a) at the large surface separation $L=10 d$. For a given binding energy $U$, different values of $X_{\mathrm{s}}$ in Fig. 10 result from different values of the chemical potential $\mu$ of the particles. The three curves shown in Fig. 10 fall onto a single curve since the steric interactions between the two adsorbed layers of particles that lead to the potential barrier only depend on the concentrations of the particles in these layers.

\section{Adsorption free energies}

In the grand-canonical ensemble, particle concentrations can be expressed as derivatives of the grand-canonical potential, or "free energy" with respect to the chemical potential $\mu$. The concentration profile $X(z)$ thus can be related to a $z$-dependent grandcanonical potential $f(z)$ via $X(z)=-\partial f(z) / \partial \mu$, and the surface concentrations $X_{\mathrm{s}}$ defined in eqn (3) can be associated with a surface potential, or "free energy" of adsorption $f_{\mathrm{s}}$. In the previous section, we have shown that the surface concentrations $X_{\mathrm{s}}$ at the surface separation $L=d$ and at large separations depend in good approximation on a single parameter, the rescaled chemical potential $\mu+U_{L}$ with $U_{L}=2 U$ for $L=d$ and 
Table 1 Surface concentrations $X_{\mathrm{s}}$ at the values $\mu^{*}$ of the chemical potential that maximize the effective binding energy $U_{\text {ef }}$ (see Fig. 7)

\begin{tabular}{llll}
\hline$U[k T]$ & $\mu^{*}[k T]$ & $X_{\mathrm{s}}(L=d)\left[1 / d^{2}\right]$ & $2 X_{\mathrm{s}}(L=10 d)\left[1 / d^{2}\right]$ \\
\hline 8 & $-12.24 \pm 0.03$ & $0.62 \pm 0.01$ & $0.63 \pm 0.01$ \\
10 & $-13.76 \pm 0.03$ & $0.67 \pm 0.01$ & $0.68 \pm 0.01$ \\
12 & $-15.44 \pm 0.03$ & $0.71 \pm 0.01$ & $0.71 \pm 0.01$ \\
\hline
\end{tabular}

$U_{L}=U$ for large $L$ (see Fig. 9). Therefore, we consider here a free energy of adsorption $f_{\mathrm{s}}\left(\mu+U_{L}\right)$ that depends on $\mu+U_{L}$ and is defined via $X_{\mathrm{s}}\left(\mu+U_{L}\right)=-\partial f_{\mathrm{s}}\left(\mu+U_{L}\right) / \partial \mu$, or alternatively via

$$
f_{\mathrm{s}}\left(\mu+U_{L}\right)=-\int X_{\mathrm{s}}\left(\mu+U_{L}\right) \mathrm{d} \mu
$$

up to an integration constant.

The free energy of adsorption $f_{\mathrm{s}}\left(\mu+U_{L}\right)$ is related to the effective, particle-mediated binding energy $U_{\text {ef }}$ via

$$
U_{\mathrm{ef}}(\mu, U) \simeq-\left(f_{\mathrm{s}}(\mu+2 U)-2 f_{\mathrm{s}}(\mu+U)\right)
$$

because $U_{\text {ef }}$ can be understood as the difference between the adsorption free energies at the binding separation $L=d$ and at large separations $L$ of the surfaces. The factor 2 in the second term on the right-hand side of eqn (5) results from the fact that we have two adsorption layers of particles at large surface separations $L$. In Fig. 7, the simple model based on eqn (5) is compared to the Monte Carlo data. The function $f_{\mathrm{s}}\left(\mu+U_{L}\right)$ here has been obtained by integrating the dashed fitting function of Fig. 9(b), with an integration constant determined from a fit to the Monte Carlo data (see Appendix for details).

According to eqn (4), the chemical potential $\mu^{*}$ at which the effective binding energy $U_{\text {ef }}$ is maximal follows from the equation

$$
\frac{\partial U_{\mathrm{ef}}}{\partial \mu} \simeq X_{\mathrm{s}}(\mu+2 U)-2 X_{\mathrm{s}}(\mu+U)=0
$$

An interesting consequence of eqn (4) thus is that we have

$$
X_{\mathrm{s}}\left(\mu^{*}+2 U\right) \simeq 2 X_{\mathrm{s}}\left(\mu^{*}+U\right)
$$

at $\mu=\mu^{*}$, i.e. the surface concentration $X_{\mathrm{s}}$ of particles at the binding separation $L=d$ is twice the surface concentration $X_{\mathrm{s}}$ at large separations $L$. Within the numerical accuracy, this is indeed the case for our MC results at the binding energies $U=8,10$, and $12 k T$ (see Table 1). The location $\mu=\mu^{*}$ of the maximum of the effective binding energy $U_{\text {ef }}(\mu)$ obtained from eqn (7) is in a good agreement with Monte Carlo results for particle binding energies $U \geq 7 k T$ (see Fig. 8). The deviation at the smaller binding energy $U=6 k T$ presumably results from contributions of the binding entropies of the particles, which are neglected in the simple model based on eqn (5). For binding energies $U \leq 5 k T$ of the particles, the effective binding energy $U_{\text {ef }}$ determined from Monte Carlo simulations does not exhibit a maximum at an intermediate value $\mu^{*}$ of the chemical potential.

\section{Generalization to other adsorption isotherms}

Our arguments in the previous section can be generalized to adsorption scenarios with particle-surface interactions $V_{\mathrm{ps}}$ and particle-particle interactions $V_{\mathrm{pp}}$ different from our simulation model, provided the particles adsorb in single layers in these scenarios, with binding energies $U$ that are significantly larger than the thermal energy $k T$. Adsorption scenarios are typically characterized by adsorption isotherms, i.e. by the surface concentration $X_{\mathrm{s}}$ of adsorbed particles as a function of the bulk concentration $X_{\mathrm{b}}$ of the particles, or alternatively, as a function of the chemical potential $\mu$ of the particles. For binding energies $U \gg k T$ of the particles, it seems plausible that $X_{\mathrm{s}}$ is a function of the rescaled chemical potential $\mu+U_{L}$ with $U_{L}=U$ for single surfaces and $U_{L}=$ $2 U$ for two surfaces with "binding separation" $L$ close to the particle diameter, as in our simulation model. In general, the adsorption isotherms $X_{\mathrm{s}}\left(\mu+U_{L}\right)$ are monotonously increasing functions, with a more or less pronounced S-shape as shown in Fig. 9(b). For such isotherms, it seems likely that there are values $\mu^{*}$ of the chemical potential that satisfy eqn (7) for given binding energies $U$, which implies that the effective, particle-mediated adhesion energy $U_{\text {ef }}$ defined in eqn (5) is maximal at these values $\mu^{*}$.

In the Langmuir adsorption scenario, for example, the particles are assumed to bind independently to "adsorption sites" at the surfaces, which leads to the surface concentration: ${ }^{17,18}$

$$
X_{\mathrm{s}}\left(\mu+U_{L}\right) \simeq \frac{1}{d^{2}} \frac{q \mathrm{e}^{\left(\mu+U_{L}\right) / k T}}{1+q \mathrm{e}^{\left(\mu+U_{L}\right) / k T}}
$$

with a numerical factor $q$ and the area $d^{2}$ per binding site for binding energies $U$ much larger than the thermal energy $k T$. Here, $d^{2} X_{\mathrm{s}}$ simply is the probability that a binding site is occupied by a particle. The surface concentration $X_{\mathrm{s}}$ in the Langmuir model 'saturates' for large values of $\mu+U_{L}$, i.e. it tends towards the limiting value $1 / d^{2}$, in contrast to the surface concentration $X_{\mathrm{s}}$ of the soft particles in the model considered here, which increases proportional to $\left(\mu+U_{L}\right)^{1 / 6}$ for large values of $\mu+U_{L}$ according to a scaling argument (see Appendix). From eqn (4) and (5), we obtain the Langmuir free energy of adsorption

$$
f_{\mathrm{s}} \simeq-\frac{k T}{d^{2}} \ln \left(1+q \mathrm{e}^{\left(\mu+U_{L}\right) / k T}\right)
$$

and the effective, particle-mediated adhesion energy

$$
U_{\mathrm{ef}} \simeq \frac{k T}{d^{2}} \ln \frac{1+q \mathrm{e}^{(\mu+2 U) / k T}}{\left(1+q \mathrm{e}^{(\mu+U) / k T}\right)^{2}}
$$

which is maximal at the value

$$
\mu^{*} \simeq-U-k T \ln q
$$

of the chemical potential.

\section{Discussion and conclusions}

In this article, we have derived a general relationship between the surface concentration, or adsorption isotherm, $X_{\mathrm{s}}$, of adsorbed particles and the effective, particle-mediated adhesion energy $U_{\text {ef }}$ of two surfaces that are bound together by the adsorbed particles. The derivation of this relationship is based on a detailed analysis of Monte Carlo results. Our main results are:

(1) The surface concentration $X_{\mathrm{s}}$ of the adsorbed particles depends in good approximation on the single parameter $\mu+U_{L}$ with $U_{L}=2 U$ in the bound state of the surfaces and $U_{L}=U$ in the unbound state, for binding energies $U$ that are large compared to the thermal energy $U$ (see Fig. 9). An integration of 
the adsorption isotherms $X_{\mathrm{s}}\left(\mu+U_{L}\right)$ leads to free energies of adsorption $f_{\mathrm{s}}\left(\mu+U_{L}\right)$ (see eqn (4)).

(2) The effective, particle-mediated adhesion energy $U_{\text {ef }}$ of the surfaces can be calculated as a difference of adsorption free energies $f_{\mathrm{s}}(\mu+2 U)$ and $2 f_{\mathrm{s}}(\mu+U)$ in the bound and unbound state of the surfaces (see eqn (5)). This calculation is in good agreement with values for the effective adhesion energy determined from the pressure on the surfaces measured in our Monte Carlo simulations (see Fig. 7).

(3) The effective adhesion energy $U_{\text {ef }}$ is maximal at an intermediate value $\mu^{*}$ of the chemical potential. This intermediate value follows from eqn (7) for the adsorption isotherms $X_{\mathrm{s}}(\mu+$ $2 U)$ and $X_{\mathrm{s}}(\mu+U)$ in the bound and unbound state of the surfaces (see Fig. 8).

(4) At the optimal chemical potential $\mu^{*}$ for adhesion, the surface concentration in the bound state of the surfaces is twice the surface concentration in the unbound state. This is a direct consequence of eqn (7).

In our model, the general relationship between the adsorption isotherm $X_{\mathrm{s}}$ of the particles and the effective adhesion energy $U_{\mathrm{ef}}$ of the surfaces described by eqn (4) and (5) holds for binding energies $U \geq 7 k T$ of the particles (see Fig. 8). For these binding energies, the differences between the binding entropies of the particles in the bound and unbound state of the surfaces apparently can be neglected. These differences arise since particles bound to both surfaces experience the superposition $V_{\mathrm{ps}}(z)+$ $V_{\mathrm{ps}}(L-z)$ of particle-surface interaction potentials, which has a different shape than the potential $V_{\mathrm{ps}}$ experienced by a particle bound to one of the surfaces. The threshold for the particle binding energies $U$ beyond which eqn (4) and (5) hold may be different for other particle-surface interaction potentials $V_{\mathrm{ps}}$, which in general will lead to other binding entropies.

At the optimal chemical potential $\mu=\mu^{*}$ for adhesion, the binding of the surfaces requires only a local rearrangement of particles since the surface concentration $X_{\mathrm{s}}\left(\mu^{*}+2 U\right)$ in the bound state of the surfaces is equal to the sum $2 X_{\mathrm{s}}\left(\mu^{*}+U\right)$ of the surface concentrations in the unbound state. At larger or smaller values of $\mu$, in contrast, the equilibration of the surface concentrations during binding requires a global transport of particles in or out of the contact zone. For large contact zones, the shear strain in the bound particle layer ${ }^{19,20}$ may impede such a transport and, thus, may lead to even larger differences between the effective adhesion energies at $\mu=\mu^{*}$ and at values of $\mu$ smaller or larger than $\mu^{*}$.

In experiments, maxima in adhesion strength have been observed for intermediate concentrations of proteins that interconnect receptors and ligands in apposing membranes, ${ }^{16,21}$ and for intermediate concentrations of nanoparticles that affect the adhesion of microparticles. ${ }^{11}$ In principle, the effective surface interactions induced by adsorbed particles can be measured directly, e.g. via the surface-force apparatus, ${ }^{12,22}$ or can be inferred from the phase behavior of colloidal systems. ${ }^{23,24}$ In colloidal systems, changes in the particle concentrations may lead to reentrant transitions in which surfaces or colloidal objects first bind with increasing concentration of adhesive particles, and unbind again when the concentration is further increased beyond the optimum concentration at which the effective adhesion energy is maximal.

In this article, we have focused on particles that exhibit purely repulsive pair interactions $V_{\mathrm{pp}}$ and a short-ranged attraction $V_{\mathrm{ps}}$ to the surfaces (see Fig. 2). However, as argued in Section 6, our general relationship between the adsorption isotherm of the particles and the effective adhesion energy of the surfaces should also hold for other particle-particle interactions $V_{\mathrm{pp}}$ or particlesurface interactions $V_{\mathrm{ps}}$ at least as long as these interactions do not lead to adsorption in multilayers. This is the case for weakly attractive particle-particle interactions $V_{\text {pp }}$ and other shortranged particle-surface interactions $V_{\mathrm{ps}}$. For more strongly attractive particle-particle interactions $V_{\mathrm{pp}}$ or long-ranged particle-surface interactions $V_{\mathrm{ps}}$ that lead to multilayers of adsorbed particles, the effective adhesion potential $V_{\text {ef }}$ will exhibit several minima that correspond to one, two, or more layers of particles between the surfaces.

Layers of particles can also arise if the bulk of particles in contact with the surfaces is quite dense, or 'liquid-like', not dilute as assumed here. Such a 'layering' has been known from computer simulation studies of 'simple' fluids composed of spherically symmetric molecules or particles that have just three translational degrees of freedom. ${ }^{25}$ Layering manifests itself as periodic oscillations of the particle concentration $X(z)$ along the normal of the surfaces, with a spacing between neighboring peaks that approximately matches the diameter of the spherical particles. The oscillations are damped as one moves away from the surfaces because the particle-surface interaction potential decays to zero with increasing distance from the substrate.

Experimentally, layering near solid surfaces can be detected as oscillations in the force profile measured with the surface forces apparatus. In this apparatus, one brings a thin film composed of, e.g. nearly spherical octamethylcyclotetrasiloxane (OMCTS) molecules between the surfaces of a pair of macroscopic cylinders coated with a thin mica sheet. ${ }^{22,26}$ The cylinders are arranged such that their axes form a right angle. By varying the distance $h$ between the cylinders, one can measure the pressure $p(h)$ exerted by the confined film on the cylinders with molecular resolution. Like the particle concentration $X(z), p(h)$ also exhibits damped oscillations with a wavelength that is equal to the bulk correlation length. ${ }^{27}$

If the confined fluid is composed of particles or molecules that also possess rotational degrees of freedom, interesting orientational effects may arise. In the case of confined liquid crystals, for example, prewetting phenomena arise at a solid surface that are driven by the precise anchoring of individual particles at the surface. ${ }^{28}$ Here, 'anchoring' refers to an energetic preference of a molecule's orientation with respect to the plane of the solid surface. In addition to these static effects, diffusion of liquid crystals in nanoconfinement is also quite unique. ${ }^{29}$

We have focused here on planar surfaces. An interesting aspect of flexible surfaces such as lipid membranes is that they can wrap around adhesive particles. ${ }^{30-33}$ A partial wrapping can lead to effective, surface-mediated interactions between the adsorbed particles $^{34-36}$ and, thus, to different adsorption isotherms of the particles, compared to planar surfaces.

\section{Appendix}

\section{Interactions}

In our model, the spherical particles are confined between two planar and parallel surfaces separated by a distance $L$ along the 
$z$-axis. The interaction potential of the particles and the surfaces is the sum of a soft repulsive and a Yukawa-like attractive term:

$$
V_{\mathrm{ps}}=U\left[a_{1}\left(\frac{d}{\hat{z}}\right)^{10}-a_{2} \frac{\exp (-\eta|\hat{z}|)}{|\hat{z}|}\right]
$$

here, $\hat{z}=z+d / 2$, and $z$ is the distance of the particle center from a surface. The parameters

$$
a_{1}=-\frac{\eta d+1}{\eta d-9} \text { and } a_{2}=-\frac{10 d \mathrm{e}^{\eta d}}{\eta d-9}
$$

are chosen such that the minimum of the potential $V_{\mathrm{ps}}$ is located at $z=d / 2$, with minimum value $-U$ where $U$ is the binding energy of the particle. The interaction range of the potential depends on the parameter $\eta$. We have chosen the value $\eta=7 d$ for which the interaction potential decays to zero at separations $z$ of the particles from the surfaces close to the particle diameter $d$ (see Fig. 2).

The particle-particle interaction is purely repulsive:

$$
V_{\mathrm{pp}}=4 k T\left(\frac{d}{r}\right)^{12}
$$

here, $r$ is the distance between the two particle centers.

\section{Pressure calculations}

In the grand-canonical ensemble, equilibrium states correspond to minima of the grand potential whose exact differential may be given as

$$
\mathrm{d} F=-S \mathrm{~d} T-N \mathrm{~d} \mu-P_{\|} L \mathrm{~d} A-P_{\mathrm{zz}} A \mathrm{~d} L
$$

where $S$ denotes entropy, $T$ is temperature, $\mu$ is the chemical potential of the particles, $A$ is the area of the surface, and $P_{\|} \equiv$ $1 / 2\left(P_{x x}+P_{y y}\right)$ and $P_{z z}$ are diagonal components of the pressure tensor $\boldsymbol{P}$. Because the particle-surface interaction depends only on distances from the surfaces in the $z$-direction, properties of our model are translationally invariant in the $x$ - and $y$-directions.

To make contact with a microscopic level of description we introduce the expression: ${ }^{25}$

$$
F=-k T \ln \Xi
$$

where $k$ is Boltzmann's constant and

$$
\Xi(T, \mu, A, L)=\sum_{N} \exp (\beta \mu N) \mathscr{Q}(T, N, A, L)
$$

is the grand-canonical partition function with $\beta=1 / k T$. In eqn (17)

$$
\mathscr{Q} \equiv \frac{\mathscr{Z}}{N ! \Lambda^{3 N}}
$$

is the partition function of the canonical ensemble for a system with $3 N$ translational degrees of freedom, $\Lambda \equiv \sqrt{\beta h^{2} / 2 \pi m}$ is the thermal de Broglie wavelength of a particle of mass $m, h$ is Planck's constant, and

$$
\mathscr{Z}=\int \mathrm{d} \boldsymbol{R} \exp [-\beta V(\boldsymbol{R})]
$$

is the configuration integral with the configurational energy

$$
V(\boldsymbol{R})=\frac{1}{2} \sum_{i=1}^{N} \sum_{\substack{j=1 \\ j \neq i}}^{N} V_{\mathrm{pp}}\left(\boldsymbol{r}_{i j}\right)+\sum_{k=1}^{2} \sum_{i=1}^{N} V_{\mathrm{ps}}\left(z_{i, k}\right)
$$

Here, $\boldsymbol{R} \equiv\left\{\boldsymbol{r}_{1}, \boldsymbol{r}_{2}, \ldots, \boldsymbol{r}_{N}\right\}$ is a short-hand notation for the configuration of the $N$ particles, $\boldsymbol{r}_{i j} \equiv\left|\boldsymbol{r}_{i}-\boldsymbol{r}_{j}\right|$ is the distance between the particles $i$ and $j$, and $z_{i, 1}=z_{i}$ and $z_{i, 2}=L-z_{i}$ are the distances of particle $i$ from the two walls located at $z=0$ and $z=$ $L$, respectively.

A key quantity in this article is the pressure tensor component $P_{z z}$. From eqn (15) and (16), it is easy to verify that

$$
\begin{aligned}
P_{z z} & =\frac{k T}{A} \frac{\partial \ln \Xi}{\partial L} \\
& =\sum_{N} \frac{1}{N ! \Lambda^{3 N}}\left(\frac{\partial \mathscr{Z}}{\partial L}\right)_{T, \mu, A} \\
& =P^{\mathrm{id}}+P_{z z}^{\mathrm{p}}
\end{aligned}
$$

where the ideal-gas contribution is $P^{\mathrm{id}}=\langle N\rangle k T / V$ with $V=A L$ and $\langle\ldots\rangle$ denotes an average in the grand-canonical ensemble. The contribution from particle interactions is given by

$$
\begin{aligned}
P_{z z}^{\mathrm{p}}= & -\frac{1}{2 V} \sum_{i=1}^{N} \sum_{\substack{j=1 \\
j \neq i}}^{N}\left\langle V_{p p}^{\prime}\left(\boldsymbol{r}_{i j}\right) \boldsymbol{r}_{i j}\left(\hat{\boldsymbol{r}}_{i j} \cdot \hat{\boldsymbol{e}}_{\mathrm{z}}\right)^{2}\right\rangle-\frac{1}{2 V} \sum_{k=1}^{2} \\
& \times \sum_{i=1}^{N}\left\langle V_{p s}^{\prime}\left(z_{i, k}\right) z_{i, k}\right\rangle
\end{aligned}
$$

where the first and second terms on the right side arise because of particle-particle and particle-surface interactions, respectively, $V_{\mathrm{pp}}{ }^{\prime}=\mathrm{d} V_{\mathrm{pp}} / \mathrm{d} \boldsymbol{r}_{i j}, V_{\mathrm{ps}}{ }^{\prime}=\mathrm{d} V_{\mathrm{ps}} / \mathrm{d} z_{i}, \hat{\boldsymbol{r}}_{i j}=\boldsymbol{r}_{i j} / \boldsymbol{r}_{i j}$, and $\hat{\boldsymbol{e}}_{z}$ is a unit vector pointing along the $z$-axis. In the limit $L \rightarrow \infty$, we have $P_{z z} \rightarrow P_{\mathrm{b}}$ where the bulk pressure is given by

$$
P_{\mathrm{b}}=P^{\mathrm{id}}-\frac{1}{6 V} \sum_{i=1}^{N} \sum_{\substack{j=1 \\ j \neq i}}^{N}\left\langle V_{\mathrm{pp}}^{\prime}\left(\boldsymbol{r}_{i j}\right) \boldsymbol{r}_{i j}\right\rangle
$$

The pressure $p$ in eqn (1) that the particle exerts on the surfaces is identical to the pressure tensor component $P_{z z}$ and calculated from eqn (21) and (22) in our Monte Carlo simulations.

\section{Monte Carlo simulations}

In our Monte Carlo (MC) simulations, we numerically realize a Markov process with a limiting distribution in configuration space proportional to $\exp \{-\beta[U(\boldsymbol{R})-\mu N]-\ln N !-3 N \ln \Lambda\}$. To achieve this we employ an algorithm originally proposed by Adams for a simple Lennard-Jones fluid. ${ }^{37}$ It proceeds in a sequence of pairs of steps where particles are displaced and created or destroyed.

We refer to a MC cycle as a sequence of $N$ attempts to displace a molecule and $N$ attempted creations of new or removals of already existing molecules where $N$ is the actual number of molecules present at the beginning of a new cycle. To avoid biasing the generation of configurations, displacements and rotations as well as creation and removal are attempted with equal probability. Our simulations are based upon $6 \times 10^{3}$ cycles for equilibration followed by $10^{5}$ cycles to compute ensemble averages. To save computing time we employ a combination of a 
conventional Verlet with a link-cell neighborlist as described in Allen and Tildesley's book. ${ }^{38}$ A particle is considered as a neighbor of a reference particle if it is located within a sphere of radius $r_{N}=3.5 \mathrm{~d}$. In addition, fluid-fluid interactions are cut off beyond an intermolecular separation of $r_{\mathrm{c}}=3 d$ which we use throughout this work; no such cutoff is applied to fluid-substrate interactions.

\section{Curve fitting}

The dashed line in Fig. 9(b) represents the $9^{\text {th }}$-order polynomial fit $X_{\mathrm{s}}\left(\mu+U_{L}\right) \simeq\left(1 / d^{2}\right) \sum_{n=0}^{9} c_{n} x^{n}$ with $x=\left(\mu+U_{L}\right) / k T$ and fit parameters $c_{0}=0.513, c_{1}=0.0335, c_{2}=-0.00212, c_{3}=0.000311$, $c_{4}=-3.03 \times 10^{-5}, c_{5}=-2.914 \times 10^{-6}, c_{6}=6.42 \times 10^{-7}, c_{7}=$ $-1.13 \times 10^{-8}, c_{8}=-2.63 \times 10^{-9}$, and $c_{9}=1.13 \times 10^{-10}$.

Integration of $X_{\mathrm{s}}\left(\mu+U_{L}\right)$ leads to the adsorption free energy $f_{\mathrm{s}}\left(\mu+U_{L}\right) \simeq\left(k T / d^{2}\right)\left(c_{\text {int }}-\sum_{n=0}^{9} c_{n} x^{n+1} /(n+1)\right)$ (see eqn (4)). We have determined the value $c_{\text {int }}=-2.69$ for the integration constant from a fit of eqn (5) to the Monte Carlo data for the effective binding energy $U_{\text {ef }}$ shown in Fig. 7.

\section{Asymptotic limit of large surface concentration}

At large surface concentrations, the particles in the adsorption layers are packed in a hexagonal lattice. To determine the scaling form of the surface concentration $X_{\mathrm{s}}$ in this limit, we consider a surface area $A$ with $N$ adsorbed particles arranged in a hexagonal lattice. The adsorption energy of the particles is $E_{\text {ad }}=N\left(\mu+U_{L}\right)$, and the sum of the repulsive interactions of the particles is $E_{\text {rep }}=3 N V_{\mathrm{pp}}(r(N))=12 N(d / r(N))^{12}$ with the $N$ dependent distance $r(N)=\sqrt{2 A / N} / 3^{1 / 4}$ between neighboring particles. Minimization of the total energy $E_{\text {ad }}+E_{\text {rep }}$ with respect to the particle number $N$ leads to a particle density of $X_{\mathrm{s}} \simeq\left(0.55 / d^{2}\right)\left(\mu+U_{L}\right)^{1 / 6}$.

\section{Acknowledgements}

We would like to thank Bartosz Różycki and Marco G. Mazza for valuable comments and fruitful discussions. Financial support from the Deutsche Forschungsgemeinschaft (DFG) via the International Research Training Group 1524 "Self-Assembled Soft Matter Nano-Structures at Interfaces" is gratefully acknowledged.

\section{References}

1 S. Srivastava, A. Verma, B. L. Frankamp and V. M. Rotello, $A d v$. Mater., 2005, 17, 617-621.

2 D. Zhang, O. Neumann, H. Wang, V. M. Yuwono, A. Barhoumi,

M. Perham, J. D. Hartgerink, P. Wittung-Stafshede and N. J. Halas, Nano Lett., 2009, 9, 666-671.

3 J. C. Ang, J.-M. Lin, P. N. Yaron and J. W. White, Soft Matter, 2010, 6, 383-390.

4 B. Bharti, J. Meissner and G. H. Findenegg, Langmuir, 2011, 27, 9823-9833.
5 M. Kendall, P. Ding and K. Kendall, Nanotoxicology, 2011, 5, 55-65.

$6 \mathrm{~K}$. Wong, P. Lixon, F. Lafuma, P. Lindner, O. Charriol and B. Cabane, J. Colloid Interface Sci., 1992, 153, 55-72.

7 R. Shenhar, T. B. Norsten and V. M. Rotello, Adv. Mater., 2005, 17, 657-669.

8 R. Sczech and H. Riegler, J. Colloid Interface Sci., 2006, 301, 376385.

9 Y. Ofir, B. Samanta and V. M. Rotello, Chem. Soc. Rev., 2008, 37, 1814-1825.

10 D. Babayan, C. Chassenieux, F. Lafuma, L. Ventelon and J. Hernandez, Langmuir, 2010, 26, 2279-2287.

11 C. Hodges, Y. Ding and S. Biggs, Adv. Powder Technol., 2010, 21, $13-$ 18.

12 Y. Hu, I. Doudevski, D. Wood, M. Moscarello, C. Husted, C. Genain, J. A. Zasadzinski and J. Israelachvili, Proc. Natl. Acad. Sci. U. S. A., 2004, 101, 13466-13471.

13 Y. Min, K. Kristiansen, J. M. Boggs, C. Husted, J. A. Zasadzinski and J. Israelachvili, Proc. Natl. Acad. Sci. U. S. A., 2009, 106, 3154-3159.

14 T. Franke, R. Lipowsky and W. Helfrich, Europhys. Lett., 2006, 76, 339-345.

15 A. Saterbak and D. A. Lauffenburger, Biotechnol. Prog., 1996, 12, 682-699.

16 M. L. Dustin, T. Starr, D. Coombs, G. R. Majeau, W. Meier, P. S. Hochman, A. Douglass, R. Vale, B. Goldstein and A. Whitty, J. Biol. Chem., 2007, 282, 34748-34757.

17 B. Różycki, R. Lipowsky and T. R. Weikl, Europhys. Lett., 2008, 84, 26004.

18 B. Różycki, R. Lipowsky and T. R. Weikl, J. Stat. Mech.: Theory Exp., 2009, P11006.

19 P. Bordarier, M. Schoen and A. Fuchs, Phys. Rev. E: Stat. Phys., Plasmas, Fluids, Relat. Interdiscip. Top., 1998, 57, 1621-1635.

20 M. Cates, J. Wittmer, J. Bouchaud and P. Claudin, Phys. Rev. Lett., 1998, 81, 1841-1844.

21 R. N. Gutenkunst, D. Coombs, T. Starr, M. L. Dustin and B. Goldstein, PLoS One, 2011, 6, e19701.

22 J. N. Israelachvili, Intermolecular and Surface Forces, Academic Press, 2nd edn, 1992.

23 M. M. Baksh, M. Jaros and J. T. Groves, Nature, 2004, 427, 139141.

24 E. M. Winter and J. T. Groves, Anal. Chem., 2006, 78, 174-180.

25 M. Schoen and S. H. L. Klapp, Nanoconfined Fluids: Soft Matter Between two and three Dimensions, Wiley-VCH, Hoboken, 2007.

26 M. L. Gee, P. M. McGuiggan, J. N. Israelachvili and A. M. Homola, J. Chem. Phys., 1990, 93, 1895-1906.

27 S. H. L. Klapp, Y. Zeng, D. Qu and R. von Klitzing, Phys. Rev. Lett., 2008, 100, 118303.

28 M. Greschek and M. Schoen, J. Chem. Phys., 2011, 135, 204702.

29 M. G. Mazza, M. Greschek, R. Valiullin, J. Kaerger and M. Schoen, Phys. Rev. Lett., 2010, 105, 227802.

30 R. Lipowsky and H. G. Döbereiner, Europhys. Lett., 1998, 43, 219 225.

31 M. Deserno, Phys. Rev. E: Stat., Nonlinear, Soft Matter Phys., 2004, 69, 031903 .

32 C. Fleck and R. Netz, Europhys. Lett., 2004, 67, 314-320.

33 S. Cao, G. Wei and J. Z. Y. Chen, Phys. Rev. E: Stat., Nonlinear, Soft Matter Phys., 2011, 84, 050901.

34 T. R. Weikl, Eur. Phys. J. E: Soft Matter Biol. Phys., 2003, 12, 265 273.

35 M. M. Müller, M. Deserno and J. Guven, Europhys. Lett., 2005, 69, 482-488.

36 B. J. Reynwar, G. Illya, V. A. Harmandaris, M. M. Müller, K. Kremer and M. Deserno, Nature, 2007, 447, 461-464.

37 D. J. Adams, Mol. Phys., 1975, 29, 307-311.

38 M. P. Allen and D. J. Tildesley, Computer Simulation of Liquids, Clarendon Press, Oxford, 1987. 